Check for updates

Cite this: J. Mater. Chem. A, 2020, 8 , 15875

Received 21st March 2020

Accepted 7th May 2020

DOI: $10.1039 / \mathrm{d} 0 \mathrm{ta} 03237 \mathrm{a}$

rsc.li/materials-a

\section{Nitrogen-doped phosphorene for electrocatalytic ammonia synthesis $\dagger$}

\author{
Guangrui Xu, $\dot{t}^{\text {ab }}$ Hao Li, $\dot{t}^{c}$ Abdulaziz S. R. Bati, (D) d Munkhjargal Bat-Erdene, ${ }^{d}$ \\ Md J. Nine, (D) Dusan Losic, (D) ${ }^{\text {e }}$ Yu Chen, (D) *a Joseph G. Shapter, (D)*d \\ Munkhbayar Batmunkh (D)*df and Tianyi Ma (D)*b
}

\begin{abstract}
The rapid surface oxidation of phosphorene under ambient conditions is considered to be a serious issue for many applications, but is used here as a strategy to achieve efficient heteroatom doping. Highly crystalline nitrogen-doped phosphorene ( $\mathrm{N}$-phosphorene) is prepared using a combination of ball milling and microwave techniques. The prepared $\mathrm{N}$-doped phosphorene nanosheets showed outstanding electrocatalytic performance as a new type of non-metallic catalyst for nitrogen $\left(\mathrm{N}_{2}\right)$ to ammonia $\left(\mathrm{NH}_{3}\right)$ conversion, with an $\mathrm{NH}_{3}$ yield rate and faradaic efficiency (FE) of up to $18.79 \mu \mathrm{g} \mathrm{h}^{-1} \mathrm{mgCAT}^{-1}$ and $21.51 \%$, respectively, at a low overpotential $(0 \mathrm{~V})$ versus the reversible hydrogen electrode (RHE). Density functional theory calculations revealed that the high nitrogen reduction reaction (NRR) FEs originate from the increased hydrophobicity at the $\mathrm{N}$ and $\mathrm{O}$ doped phosphorene surfaces, which in turn hinders the competing hydrogen evolution reaction (HER) in an alkaline environment and promotes the NRR. This work not only introduces an efficient strategy to chemically functionalize 2D phosphorene, but also opens a new avenue in using $\mathrm{N}$-doped phosphorene nanosheets as a metal-free catalyst.
\end{abstract}

\section{Introduction}

Atomically thin two-dimensional (2D) layered nanomaterials have emerged as one key component to future materials development. Since 2014, black phosphorus (BP) - a layered 2D semiconductor - has attracted increasing attention due to its fascinating properties and unique structure. ${ }^{1}$ Phosphorene, mono- or few-layered BP obtained from bulk BP, exhibits a thickness-dependent tunable bandgap (0.3-2.0 eV) and excellent carrier mobility $\left(\sim 1000 \mathrm{~cm}^{2} \mathrm{~V}^{-1} \mathrm{~s}^{-1}\right)$ in addition to its high in-plane anisotropic properties,,$^{2-4}$ all of which make it attractive for a wide range of practical applications including electronic devices, ${ }^{5}$ energy storage, ${ }^{6}$ solar cells, ${ }^{7}$ and catalysis. ${ }^{8}$ Owing to its high carrier mobility, favorable electronic structure

${ }^{a}$ Key Laboratory of Macromolecular Science of Shaanxi Province, School of Materials Science and Engineering, Shaanxi Normal University, Xi'an 710062, China. E-mail: ndchenyu@gmail.com

${ }^{b}$ School of Environmental and Life Sciences, The University of Newcastle (UON), Callaghan, New South Wales 2308, Australia. E-mail: tianyi.ma@newcastle.edu.au

'Department of Chemistry, The University of Texas at Austin, Austin, TX, 78712, USA

${ }^{d}$ Australian Institute for Bioengineering and Nanotechnology, The University of Queensland, Brisbane, Queensland 4072, Australia. E-mail: j.shapter@uq.edu.au

${ }^{e}$ School of Chemical Engineering and Advanced Materials, The University of Adelaide, Adelaide, South Australia 5005, Australia

${ }^{f}$ Centre for Clean Environment and Energy, Griffith University, Gold Coast, Queensland 4222, Australia. E-mail: m.batmunkh@griffith.edu.au

$\dagger$ Electronic supplementary information (ESI) available. See DOI: 10.1039/d0ta03237a

\$ These two authors made an equal contribution to this work. and large specific surface area, BP derivatives hold specific promise for both photocatalytic and electrocatalytic processes. ${ }^{9-11}$ Although excellent progress has been made using $\mathrm{BP}$ derivatives for the hydrogen and oxygen evolution reactions, ${ }^{\mathbf{1 0 - 1 5}}$ practical demonstration of the use of $2 \mathrm{D}$ phosphorene for advanced catalysis for the nitrogen reduction reaction (NRR) and $\mathrm{CO}_{2}$ reduction reaction is very scarce. ${ }^{16}$ As such, developing efficient metal-free catalysts for these cutting-edge catalytic processes is of great interest.

Few-layer phosphorene nanosheets tend to oxidize in the presence of ambient air and water, resulting in the rapid loss of their semiconducting properties. ${ }^{17,18}$ Although this high chemical reactivity of phosphorene with oxygen is typically considered undesirable for device applications, it may also become a broad utility for the chemistry of $2 \mathrm{D}$ phosphorene. Chemical functionalization has proven to be an effective strategy in modulating the chemical, electronic, electrical and structural properties of $\mathrm{BP}$, while producing stable phosphorene nanosheets. ${ }^{\mathbf{1 9 - 2 1}}$ In particular, elemental doping is known to be a reliable approach to modify the charge carrier concentration, thus modifying the material's properties. However, there has been limited effort in the elemental doping of 2D BP despite several theoretical studies suggesting great promise..$^{22-24}$ Notably, a number of successful studies on doping of some metal elements ( $\mathrm{K}$, As, Se, and Te) into bulk BP crystals and/or mechanically exfoliated BP nanosheets have been reported,,$^{\mathbf{2 0 2 5 - 2 7}}$ while non-metal element doping and solution processability of phosphorene are areas of great interest but have not progressed yet. 
Herein, we present a facile and efficient strategy based on a combination of ball milling and microwave exfoliations to produce $\mathrm{N}$-doped phosphorene (N-phosphorene) nanosheets and demonstrate their application as a novel metal-free electrocatalyst for electrochemical nitrogen-to-ammonia $\left(\mathrm{N}_{2}\right.$ to $\mathrm{NH}_{3}$ ) synthesis under ambient conditions. Although the oxidation of BP is considered to be a significant issue, we elegantly used it to our advantage, providing abundant reactive sites for $\mathrm{N}$-doping of phosphorene. Our N-phosphorene electrocatalyst exhibited excellent electrochemical performance with high $\mathrm{NH}_{3}$ yield rates and faradaic efficiencies (FEs) of $18.79 \mu \mathrm{g} \mathrm{h} \mathrm{h}^{-1}$ $\mathrm{mg}_{\mathrm{CAT}}{ }^{-1}$ and $21.51 \%$ (determined using the indophenol blue method) and $16.67 \mu \mathrm{g} \mathrm{h}^{-1} \mathrm{mg}_{\mathrm{CAT}}{ }^{-1}$ and $19.08 \%$ (determined using an ammonia selective electrode), respectively, at a low

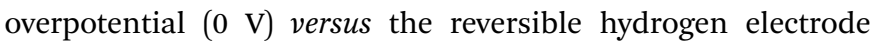
(RHE). The catalytic activity of functionalized phosphorene nanosheets has been systematically investigated using density functional theory (DFT) calculations.

\section{Results and discussion}

\section{Synthesis and characterization of $\mathbf{N}$-doped phosphorene}

2D N-phosphorene sheets were prepared in $\mathrm{N}$-methyl-2-pyrrolidone (NMP) solvent using a combination of ball milling and microwave-assisted exfoliation methods. The synthetic procedure of the N-phosphorene sheets is shown in Fig. 1a. It is well established that for chemical doping of $2 \mathrm{D}$ graphene and carbon materials, oxygen containing functional groups on the surface are responsible for the formation of a $\mathrm{C}-\mathrm{N}$
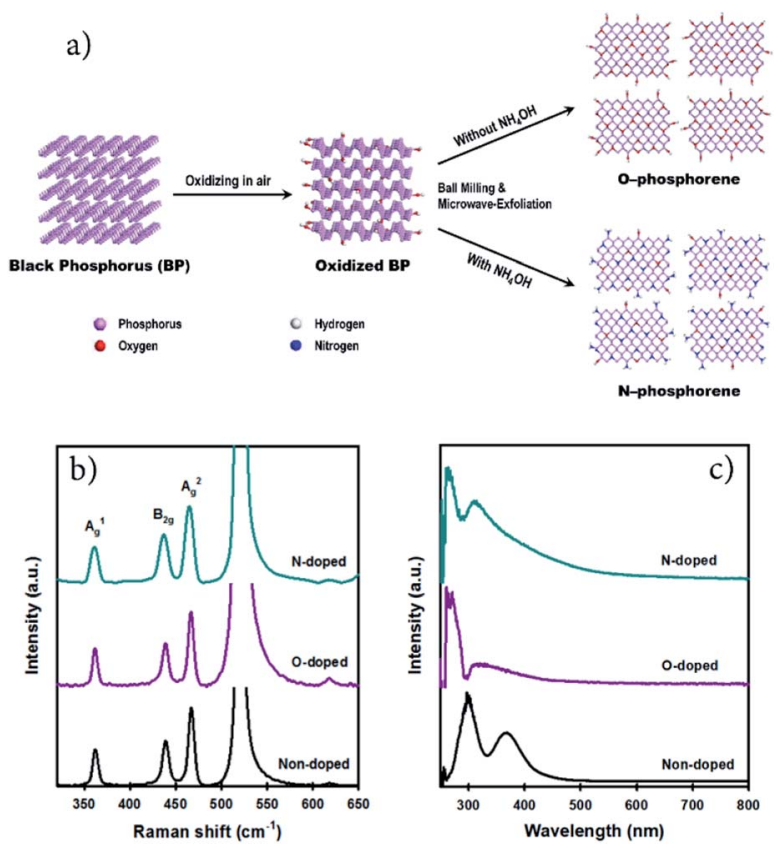

Fig. 1 (a) Schematic illustration for the preparation of $\mathrm{N}$-doped and $\mathrm{O}$-doped phosphorene using a combination of ball milling and microwave-assisted LPE methods. (b) Raman (on a silicon (Si) substrate) and (c) UV-vis spectra of non-doped, O-doped and $\mathrm{N}$ doped phosphorene. bond (N-doping). ${ }^{28-30}$ Considering this, a bulk BP crystal was first ground in a mortar and pestle in air, creating abundant reactive sites on the surface to enhance its interaction with the $\mathrm{N}$ source. The ground $\mathrm{BP}$ crystal was then ball milled in NMP solvent with and without ammonia solution $\left(\mathrm{NH}_{4} \mathrm{OH}\right)$ as the $\mathrm{N}$ source to produce $\mathrm{N}$-doped ( $\mathrm{N}$-phosphorene) and oxidized phosphorene (O-phosphorene), respectively.

Ball milling is an effective approach for edge functionalization of $2 \mathrm{D}$ layered materials, generating abundant reactive species (radicals and ions) at the nanosheet edges. ${ }^{31-33}$ In the presence of the $\mathrm{N}$ source $\left(\mathrm{NH}_{4} \mathrm{OH}\right)$, the oxidized BP flakes can be $\mathrm{N}$-doped and/or $\mathrm{N}$-functionalized during the ball-milling process (Fig. 1a) with a slight preference at the flake edges if the O-phosphorene behaves as other 2D materials during ballmilling. Notably, ball milling BP flakes in the absence of $\mathrm{NH}_{4} \mathrm{OH}$ produced highly oxidized and/or oxygen doped phosphorene nanosheets (termed "O-phosphorene"). In addition, the van der Waals interaction between the flakes may be weakened during the ball milling, producing readily exfoliatable BP flakes. Then the ball milled BP flakes were further exfoliated in NMP using a microwave-assisted liquid-phase exfoliation (LPE) method to produce ultrathin (few-layer) phosphorene nanosheets. For comparison, we also prepared few-layer phosphorene nanosheets with controlled oxidation (termed "phosphorene") using a microwave-exfoliation method (air oxidation and ball milling steps were not involved) using the procedure reported in our recent work. ${ }^{34}$

As depicted in Fig. S1a, $\dagger$ the height of the non-doped phosphorene nanosheet was measured to be $\sim 4.9 \mathrm{~nm}$, which (considering that monolayer phosphorene is $0.85 \mathrm{~nm}$ thick) ${ }^{35,36}$ corresponds to about 5 atomic layers and is consistent with our previous measurements. ${ }^{34}$ The thickness of the O-phosphorene was $\sim 5.5 \mathrm{~nm}$ (6 layers) (Fig. S1b $\dagger$ ), while the measured height for the N-phosphorene was $\sim 8.6 \mathrm{~nm}$ (10 layers) (Fig. S1c $\dagger$ ). The thicker nanosheets of our N-phosphorene, as compared to the non-doped phosphorene, could be due to the presence of functional groups and doping elements increasing the measured AFM height, ${ }^{37}$ and/or acting as a barrier during the exfoliation. Another noticeable feature from the AFM images was the difference in the flake sizes of the samples. In particular, the lateral sizes of both O-doped and N-doped phosphorene sheets varied from $150 \mathrm{~nm}$ to $300 \mathrm{~nm}$, while the non-doped phosphorene showed an average flake size of $600 \mathrm{~nm}$. This significant difference in the lateral size of the non-doped and doped phosphorene nanosheets can be explained by the ball milling induced mechanochemical process. It is well established that ball milling 2D materials such as graphite results in a dramatic reduction in the flake size, but it generates reactive sites which can facilitate the doping and functionalization. ${ }^{31,32}$ Fig. 1b shows the Raman spectra of our non-doped and doped phosphorene on a silicon ( $\mathrm{Si}$ ) substrate. The characteristic $\mathrm{A}_{\mathrm{g}}^{1}, \mathrm{~B}_{2 \mathrm{~g}}$, and $\mathrm{A}_{\mathrm{g}}^{2}$ phonon modes centered at $\sim 362, \sim 437$, and $\sim 466 \mathrm{~cm}^{-1}$, respectively, ${ }^{17,38}$ were observed for all three samples, suggesting that the chemical doping/functionalization and ball milling treatments did not alter the structural integrity. Moreover, our Raman spectra are consistent with the signature 
Raman spectrum of partially oxidized few-layer black phosphorus. $^{39}$

We further examined our non-doped and doped phosphorene dispersions using UV-vis spectroscopy (Fig. 1c). Two main absorption peaks can be observed for all samples and are consistent with previously reported theoretical results and the spectrum of mechanically exfoliated $\mathrm{BP} .^{\mathbf{4 0 , 4 1}}$ In particular, the non-doped phosphorene dispersion exhibited peaks at around $300 \mathrm{~nm}$ and $370 \mathrm{~nm}$, which are in excellent agreement with our previous study on microwave-exfoliated few-layer BP solution. ${ }^{34}$ Interestingly, despite the appearance of these two peaks, considerable shifts can be observed for the $\mathrm{O}$ - and $\mathrm{N}$-doped phosphorene samples, as compared to the non-doped phosphorene (Fig. 1c). Recently, we used DFT calculations to determine the UV-vis absorbance of phosphorene, ${ }^{34}$ and observed the two characteristic peaks within the range 250-400 $\mathrm{nm}$ (Fig. S2 $\dagger$ ). More importantly, we theoretically found that the positions of the spectral peaks can shift depending on the BP flake size (blueshift as the flakes become smaller). ${ }^{34}$ Owing to the small flake sizes (confirmed by AFM), the peak positions of our doped phosphorene samples blueshifted to $260 \mathrm{~nm}$ and $310 \mathrm{~nm}$, which qualitatively agree with the simulated UV-vis spectrum of the small phosphorene sheet with high accuracy (Fig. S2 $\dagger$ ). In addition, as shown in Fig. S3, $\dagger$ our N-phosphorene dispersion showed excellent stability over at least 20 days.

$\mathrm{X}$-ray photoelectron spectroscopy (XPS) is an important tool to characterize the chemical structure of nanostructured functional materials. In this work, we used XPS to explore the oxidation and functionalization/doping states of our phosphorene samples. Fig. 2a-c show the high-resolution (HR) P 2p XPS spectra of our non-doped, O-doped and N-doped phosphorene nanosheets. All phosphorene samples show the $\mathrm{P} 2 \mathrm{p}_{3 / 2}$ and $\mathrm{P} 2 \mathrm{p}_{1 / 2}$ doublet at around 129.9 and $130.8 \mathrm{eV}$, respectively, which can be assigned to the signals of $\mathrm{P}-\mathrm{P}$ bonds. ${ }^{19}$ In addition, broad peaks at around $134.4 \mathrm{eV}$, corresponding to $\mathrm{P}-\mathrm{O}$ bonds $\left(\mathrm{P}_{x} \mathrm{O}_{y}\right),{ }^{10}$ can be observed for all samples. However, significant differences in the intensity of these peaks associated with $\mathrm{P}_{x} \mathrm{O}_{y}$ can be used to evaluate the degree of oxidation. As expected, only slight oxidation was observed for the non-doped phosphorene nanosheets (Fig. 2a) as no ball milling or air oxidation steps were involved in the sample preparation. Moreover, all experimental steps except the microwaveexfoliation were carried out in a $\mathrm{N}_{2}$-filled glovebox to control the oxidation. As shown in Fig. 2b, the O-phosphorene exhibited an intense $\mathrm{P}_{x} \mathrm{O}_{y}$ peak centered at $134.4 \mathrm{eV}$, confirming the strong oxygen doping and/or functionalization of BP. Due to its tendency to oxidize in ambient air, the as-produced phosphorene nanosheets were heavily oxidized during the air oxidation and ball milling in the absence of any doping agent. Interestingly, when the doping agent $\left(\mathrm{NH}_{4} \mathrm{OH}\right)$ was used during the ball milling, the peak at higher binding energy (131.2-136.0 eV) broadened, in addition to the existence of two signals from $\mathrm{P}-\mathrm{P}$ bonds (Fig. 2c). The peak can be deconvoluted into three main spin-orbit split doublets with a splitting of $0.86 \mathrm{eV}$. While the $\mathrm{P}$ $2 \mathrm{p}_{3 / 2}$ peaks centered at around $129.8 \mathrm{eV}$ and $134.3 \mathrm{eV}$ can be assigned to $\mathrm{P}-\mathrm{P}$ and $\mathrm{P}_{x} \mathrm{O}_{y}$, the extra peak at $\sim 133 \mathrm{eV}$ is attributed to the $\mathrm{P}-\mathrm{N}$ bonds. ${ }^{21,33,42}$ More importantly, the N-phosphorene sample shows lower $\mathrm{P}_{x} \mathrm{O}_{y}$ peak intensity as compared to the O-phosphorene, suggesting that some of the oxygen containing groups were replaced by nitrogen functional groups during ball milling. Ball milling is an effective method not only for functionalizing graphene nanosheets; ${ }^{31,32}$ it was also found to be a powerful strategy to form stable phosphorus-carbon (P-C) bonds in a BP and graphite composite. ${ }^{43}$ The successful Ndoping was further corroborated by the XPS survey scan (Fig. S4 $\dagger$ ) and HR N 1s spectrum (Fig. 2d) of the N-phosphorene sample.

We further characterized our N-phosphorene sample using transmission electron microscopy (TEM). The TEM image presented in Fig. 2e shows that the flake size of our N-phosphorene nanosheets is around $200 \mathrm{~nm}$, which is in agreement with the lateral sizes measured from the AFM images (Fig. S1c $\dagger$ ) and UVvis absorption spectrum of a small phosphorene nanosheet (Fig. S2 $\dagger$ ). As depicted in the inset of Fig. 2f, the selected area
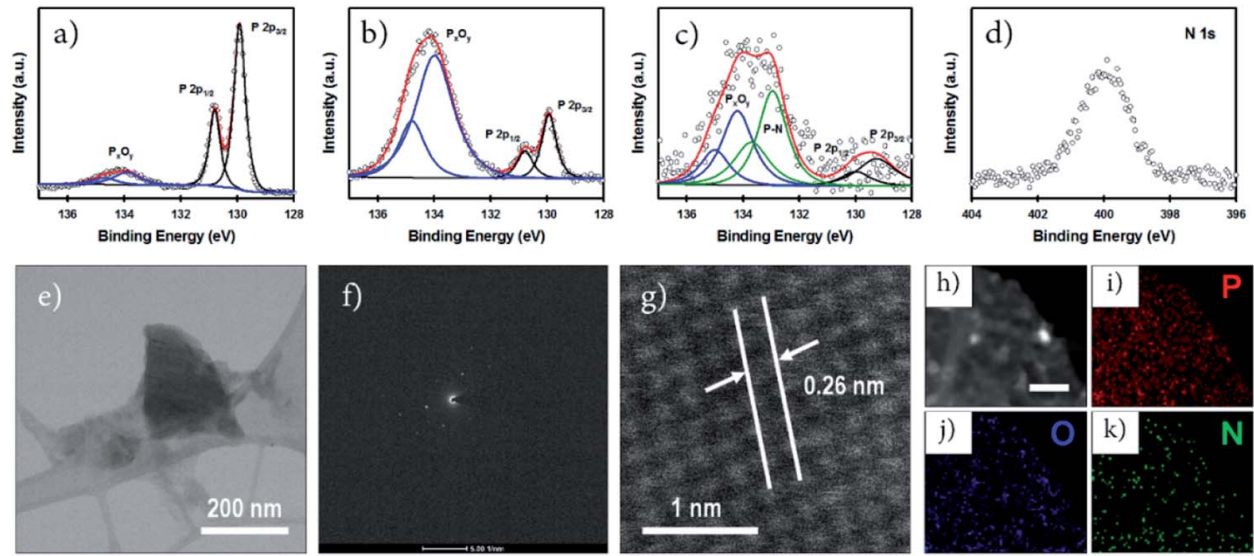

Fig. 2 High resolution P 2p XPS spectra of (a) non-doped, (b) O-doped, and (c) N-doped phosphorene nanosheets. (d) N 1s XPS spectrum, (e) TEM image, (f) SAED pattern and (g) HRTEM image of the N-phosphorene. (h) High-angle annular dark-field-scanning transmission electron microscope (HAADF-STEM) image of an $\mathrm{N}$-phosphorene nanosheet (scale bar: $50 \mathrm{~nm}$ ), and (i-k) the corresponding EDX elemental mapping images. 
electron diffraction (SAED) pattern of our N-phosphorene confirms the high-quality single-crystal structure of BP, and is also consistent with a previous report of exfoliated BP. ${ }^{44}$ The measured lattice spacing $(0.26 \mathrm{~nm})$ of our $\mathrm{N}$-phosphorene is in excellent agreement with previous literature (Fig. 2g). ${ }^{9,11}$ As shown in Fig. S5, $\uparrow$ ultrasmall atomic size dots (white) can be observed in the HRTEM images of the N-phosphorene nanosheet, particularly at the edge (as expected), ${ }^{32}$ which could be due to the significant functionalization and doping. Furthermore, the successful $\mathrm{N}$-doping and the presence of each element $(\mathrm{P}, \mathrm{O}$ and $\mathrm{N})$ in our $\mathrm{N}$-phosphorene sample was confirmed by energy-dispersive X-ray spectroscopy (EDX) elemental mapping (Fig. 2h-k).

\section{Electroreduction of $\mathbf{N}_{2}$ to $\mathrm{NH}_{3}$}

The NRR electrocatalytic activity of all catalysts was evaluated in an $\mathrm{N}_{2}$-saturated $0.1 \mathrm{M} \mathrm{KOH}$ solution. Linear sweep voltammograms (LSVs) of N-phosphorene were recorded in $0.1 \mathrm{M} \mathrm{KOH}$ solution saturated with Ar or $\mathrm{N}_{2}$ (Fig. 3a). It can be seen that the measured current density in $\mathrm{N}_{2}$-saturated solution was higher than that of the Ar-saturated solution when using $\mathrm{N}$ phosphorene as the cathodic catalyst, indicating the effective catalysis of the NRR using N-phosphorene. The NRR occurred at a potential of $+0.05 \mathrm{~V}$, which was close to the equilibrium
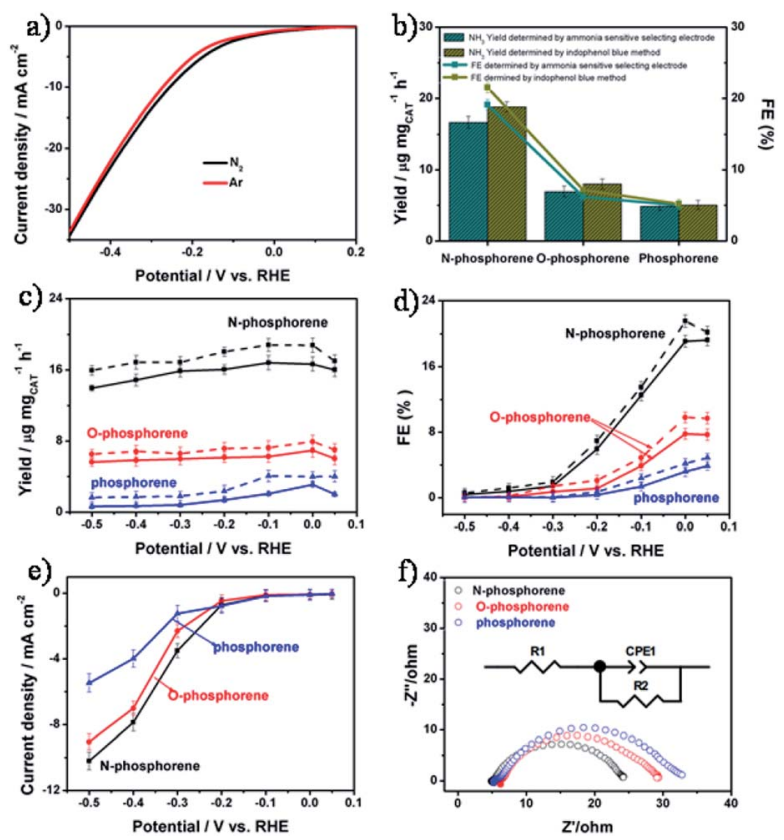

Fig. 3 (a) LSV curves of $\mathrm{N}$-phosphorene in $\mathrm{Ar}$ - and $\mathrm{N}_{2}$-saturated $0.1 \mathrm{M}$ $\mathrm{KOH}$ measured with a scan rate of $5 \mathrm{mV} \mathrm{s}^{-1}$. (b) Yield and FE of $\mathrm{N}$ phosphorene, O-phosphorene and phosphorene determined using two different methods (ammonia selective electrode method and indophenol blue method). (c) $\mathrm{NH}_{3}$ yield rates and (d) FEs of the three catalysts at different potentials ranging from $+0.05 \mathrm{~V}$ to $-0.5 \mathrm{~V}$ determined using the ammonia selective electrode (solid line) and the indophenol blue method (dashed line), and (e) the corresponding current densities. (f) EIS spectra at an applied potential of $0 \mathrm{~V} v \mathrm{vs}$. RHE of the three electrocatalysts, and their corresponding equivalent-circuit diagram. thermodynamic potential ( $+0.533 \mathrm{~V} v s$. RHE) for the NRR under the same conditions. The low onset potential of N-phosphorene for the NRR was similar to that of $\mathrm{Ru} @ \mathrm{ZrO}_{2} / \mathrm{NC}$ (ruthenium single atom in $\mathrm{ZrO}_{2} / \mathrm{N}$-doped porous carbon) which showed a $\mathrm{FE}$ of up to $21 \%$ at $-0.11 \mathrm{~V}^{45}$ With $\mathrm{N}_{2}$ gas bubbling into the cathodic compartment, the chronoamperometry curves of electrolysis at different potentials were measured under ambient conditions using N-phosphorene, O-phosphorene and phosphorene as the catalysts (Fig. S6a-c $\dagger$ ).

The production of $\mathrm{NH}_{3}$ was detected and quantified using two different methods, namely an ammonia selective electrode method $^{46,47}$ (Fig. S7 $\dagger$ ) and the indophenol blue method ${ }^{48,49}$ (Fig. S8 $\dagger$ ). The possible by-product $\mathrm{N}_{2} \mathrm{H}_{4}$ was detected using the Watt and Chrisp method (Fig. S9 $\dagger$ ). ${ }^{48-50}$ As shown in Fig. 3b, Nphosphorene exhibited an excellent yield rate of $16.67 \mu \mathrm{g} \mathrm{h} \mathrm{h}^{-1}$ $\mathrm{mg}_{\mathrm{CAT}}{ }^{-1}$ at $0 \mathrm{~V}$ determined using an ammonia selective electrode, which was more than three times and five times higher than that of O-phosphorene and phosphorene, respectively. The FE of $\mathrm{NH}_{3}$ generation catalyzed by $\mathrm{N}$-phosphorene was measured to be about $19.08 \%$ at $0 \mathrm{~V}$, which was significantly higher than that of the O-phosphorene (4.86\%) and phosphorene $(3.19 \%)$. The prominent catalytic activity of $\mathrm{N}$-phosphorene could be attributed to the exposure of enhanced active sites of the nanosheets as well as the electronic structure obtained through N-doping. We also used the indophenol blue method to quantify the produced $\mathrm{NH}_{3}$. UV-vis absorption spectra of the electrolytes colored with the indophenol indicator in the dark for $3 \mathrm{~h}$ are plotted in Fig. S6d-f. $\dagger$ The results show the highest absorbance intensity when electrolyzed at $0 \mathrm{~V} v s$. RHE. Although the yield rates of $\mathrm{N}$-phosphorene, O-phosphorene and phosphorene determined by the indophenol blue method were slightly higher than those determined using the ammonia selective electrode method, the same trends were observed for both methods. In particular, the yield rate for our $\mathrm{N}$ phosphorene measured using the indophenol blue method was $18.79 \mu \mathrm{g} \mathrm{h}^{-1} \mathrm{mg}_{\mathrm{CAT}}{ }^{-1}$ and its corresponding FE was $21.51 \%$ at a low overpotential $(0 \mathrm{~V})$ (see Fig. $3 \mathrm{~b})$. These values are comparable to or even higher than those of some metal catalysts reported so far. Table S1 $\dagger$ compares the NRR performance of our N-phosphorene and the state-of-the-art electrocatalysts reported in the literature. Notably, no by-product $\mathrm{N}_{2} \mathrm{H}_{4}$ was detected by the Watt and Chrisp method (Fig. S10†), suggesting good selectivity of N-phosphorene for the NRR.

To confirm the origin of the $\mathrm{NH}_{3}$ produced, several control experiments were performed. $\mathrm{No} \mathrm{NH}_{3}$ was detected in $\mathrm{Ar}-$ saturated electrolyte using $\mathrm{N}$-phosphorene on a copper foam (CF) (Fig. S11†), suggesting that the raw material for the $\mathrm{NH}_{3}$ comes from the feed gas injected in the electrolyte. Also, no $\mathrm{NH}_{3}$ was detected in $\mathrm{N}_{2}$-saturated electrolyte with a pure $\mathrm{CF}$ electrode (Fig. S12 $\dagger$ ). These results indicate that $\mathrm{NH}_{3}$ was produced using only the $\mathrm{N}_{2}$ gas injected into the electrolyte catalyzed by the electrocatalysts.

To explore the differences among the three catalysts in detail, the $\mathrm{NH}_{3}$ yield rates, FEs and current densities of $\mathrm{N}$ phosphorene, O-phosphorene and phosphorene at different potentials ranging from $+0.05 \mathrm{~V}$ to $-0.5 \mathrm{~V}$ are illustrated in Fig. 3c-e, respectively. The $\mathrm{NH}_{3}$ yield rates were measured to be 
around $15 \mu \mathrm{g} \mathrm{h}^{-1} \mathrm{mg}_{\mathrm{CAT}}{ }^{-1}$ at all potentials, but the FEs gradually reduced as the potential shifted from +0.05 to $-0.5 \mathrm{~V}$. This is due to the fact that the hydrogen evolution reaction (HER) process has gradually dominated the reaction as the potentials become more negative. Compared to the O-phosphorene and phosphorene, the $\mathrm{NH}_{3}$ yield rates and FEs of $\mathrm{N}$-phosphorene were much higher at all applied potentials while the measured current densities were roughly similar, especially at low applied potentials (Fig. 3e), confirming the better activity of $\mathrm{N}$-phosphorene for the NRR. Particularly, at potentials below $-0.4 \mathrm{~V}$, the current densities measured for all our electrocatalysts were very similar, but the N-phosphorene exhibited significantly higher FEs than the other two catalysts, verifying the significant role of N-phosphorene in suppressing the HER during the NRR at low overpotentials.

In order to gain better insights into the intrinsic activity for the NRR, electrochemical impedance spectroscopy (EIS) was used to measure the charge transfer resistance $\left(R_{\mathrm{ct}}\right)$ of the electrodes. As shown in Fig. 3f, the $R_{\mathrm{ct}}$ of N-phosphorene (24.2 $\Omega$ ) is much lower than that of O-phosphorene (34.1 $\Omega$ ) and phosphorene (56.3 $\Omega$ ), suggesting a favorable charge transfer mechanism for the $\mathrm{N}$-doped phosphorene system due to the presence of the heteroatoms.

The durability of the N-phosphorene was evaluated by performing six consecutive electrolysis cycles at a constant potential of $0 \mathrm{~V}$ (Fig. 4a). The $\mathrm{NH}_{3}$ yield rate and $\mathrm{FE}$ decreased slightly after six cycles of chronoamperometric runs, indicating the good stability of N-phosphorene during continuous NRR electrocatalysis. In addition, the long-term electrolytic test revealed that the N-phosphorene has excellent stability over time, maintaining stable current density over 15 h (Fig. 4b), which further confirmed the good stability of N-phosphorene for the NRR electrocatalysis. The inherent properties of the $\mathrm{N}$ phosphorene should be explored after the catalysis as phosphorene nanosheets are known to be unstable in water and in oxygen-enriched environments. ${ }^{\mathbf{1 6}}$ Our N-phosphorene nanosheets were characterized after six cycles of continuous NRR electrocatalysis to determine whether the structure and composition have changed during the NRR. The existence of $\mathrm{P}$, $\mathrm{O}$ and $\mathrm{N}$ in the elemental mapping of $\mathrm{N}$-phosphorene nanosheets after six cycles of continuous catalysis suggests excellent stability. As depicted in Fig. S13, $\uparrow$ the increased signal for $\mathrm{N}$ in the catalytic material can be explained by the adsorption of $\mathrm{N}_{2}$
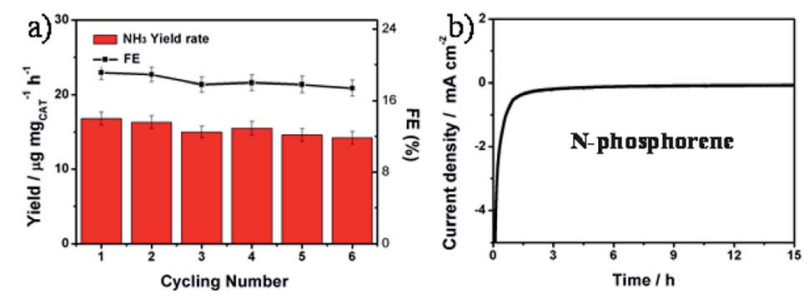

Fig. 4 (a) Cycling stability results of $\mathrm{N}$-phosphorene at $0 \mathrm{~V}$ vs. RHE. (b) Long-term durability test with chronoamperometry of $\mathrm{N}$-phosphorene toward the electrocatalytic NRR in a $\mathrm{N}_{2}$-saturated $0.1 \mathrm{M} \mathrm{KOH}$ solution for $15 \mathrm{~h}$ at $0 \mathrm{~V}$ vs. RHE. during the catalysis, which is not surprising and was expected. Furthermore, the HR P 2p XPS spectrum, shown in Fig. S14, $\dagger$ indicates that the surface composition of N-phosphorene barely changed during the electrolysis process.

As our catalyst contains $\mathrm{N}$, it is important to consider the origin of the $\mathrm{N}$ in the $\mathrm{NH}_{3}$ produced. As stated earlier, when $\mathrm{Ar}$ was bubbled through the electrolyte instead of $\mathrm{N}_{2}$, no ammonia was observed (Fig. S11 $\dagger$ ). Additionally using the XPS survey spectra, an estimate of the amount of $\mathrm{N}$ present in the catalyst can be obtained. XPS is done on oxidized Si wafers so there are for example multiple contributions to the $\mathrm{O}$ 1s peak (catalyst and substrate). We have used very conservative assumptions for the contributions to various peaks to maximize the $\mathrm{N}$ content in the catalyst. Using these assumptions, the amount of $\mathrm{N}$ present in the catalyst is between 8 and $17 \mu \mathrm{g}$. The amount of $\mathrm{N}$ produced during the durability test (in Fig. 4a) was calculated to be $22.9 \mu \mathrm{g}$ considering that the total mass of $\mathrm{NH}_{3}$ produced after six consecutive electrolysis cycles is $27.8 \mu \mathrm{g}$. Clearly all this $\mathrm{N}$ could not have come from the catalyst and in fact given the very small change in $\mathrm{NH}_{3}$ produced per hour over six cycles (6 hours), it is clear that the catalyst must make a very minor or even no contribution to the production of $\mathrm{NH}_{3}$.

\section{Theoretical investigation}

To understand the electrocatalytic results found in the experiments, DFT calculations were performed to study (i) the thermodynamic positions of $\mathrm{N}$ and $\mathrm{O}$ in a phosphorene structure, (ii) the hydrophobicity of phosphorene surfaces, and (iii) the free energy pathways of the NRR at different possible sites. Firstly, the thermodynamic doping positions of $\mathrm{N}$ and $\mathrm{O}$ were evaluated by using a multilayer phosphorene model, with the $\mathrm{N} /$ $\mathrm{O}$ doped at the upper site of the first layer as the energy reference (Fig. 5). It was found that both $\mathrm{N}$ and $\mathrm{O}$ tend to stay at the topmost surface of phosphorene. The formation energies of the $\mathrm{N}$ - and $\mathrm{O}$-doped phosphorene were also calculated (Fig. S16 $\dagger$ ). The formation of the O-doped structure has negative energy, while the formation of the N-doped structure is endothermic.

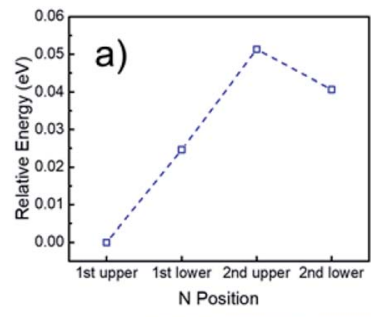

c) 909898989898989898

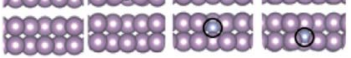

1st upper 1st lower 2nd upper 2nd lower
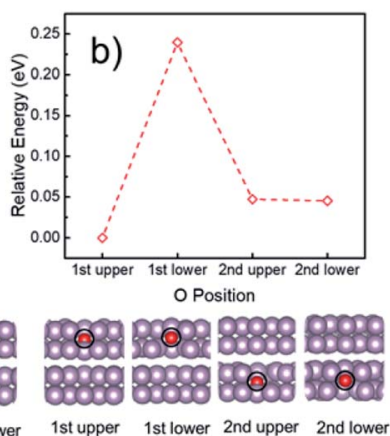

Fig. 5 Calculated relative energy of the phosphorene structure doped with (a) $\mathrm{N}$ and (b) $\mathrm{O}$ at different positions, with the optimized structures of the first two layers shown in (c). The $\mathrm{N}$ and $\mathrm{O}$ doped at the upper layer of the topmost (i.e., first) phosphorene surface were used as the energy references for (a) and (b), respectively. The complete optimized structures can be found in Fig. S15. $\uparrow$ Purple, blue, and red spheres represent $P, N$, and $O$, respectively. 
This helps to explain why there is a high amount of $\mathrm{O}$ in the Nphosphorene as found from our XPS (Fig. 2c) and EDX (Fig. 2hk) results. Nevertheless, due to the kinetically controlled synthetic method and the relatively abundant $\mathrm{N}$ and $\mathrm{O}$ after synthesis, we expect that the $\mathrm{N}$ and $\mathrm{O}$ atoms are doped in all of the layers, while the topmost layer tends to possess higher ratios of $\mathrm{N}$ and $\mathrm{O}$, as compared to other layers.

Next, DFT calculations were performed to evaluate the surface hydrophobicity by calculating the adsorption free energy of water at different sites (Fig. 6a), with the reason that water adsorption and dissociation are the most important steps that determine the HER activity in an alkaline solution. ${ }^{51}$ The pure, doped, and defected surfaces of a layered phosphorene were considered for the calculations, based on our experimental characterization (Fig. 2) and the DFT results (Fig. 5). Since the catalytic reactions are usually site-specific, ${ }^{52}$ we evaluated six typical sites at the surface of the phosphorene structure: $\mathrm{P}, \mathrm{P}-\mathrm{N}$ (P-site), P-N (N-site), P-O (P-site), P-O (O-site), and P-vacancy (Fig. 6a and b) for water and the NRR-related adsorbates. Interestingly, it is found that although a pure phosphorene surface is relatively hydrophobic already (which is slightly exothermic), the doping of $\mathrm{N}$ or $\mathrm{O}$ can further increase the hydrophobicity by having an endothermic water adsorption free energy at all $\mathrm{P}, \mathrm{N}$, and $\mathrm{O}$ sites. All the optimized adsorption geometries indicate that unlike those metallic surfaces, ${ }^{53}$ water does not form a stabilized binding geometry at all these evaluated phosphorene surfaces. This hydrophobic nature was also discovered in other p-block elements such as sulfur, by combined experimental and DFT methods. ${ }^{\mathbf{5 4 5 5}}$ The hydrophobicity found at all the doped phosphorene surfaces indicates that the HER process is relatively hindered at these surfaces, which in turn improves the FEs of the NRR. We expect that there is a similar chance for the protonation of the surface or $\mathrm{N}_{2}$ under various hydrophobicities. However, similar to previous studies on electrocatalytic oxygen reduction, ${ }^{56}$ it has been broadly discussed that surface hydrophobicity significantly influences the prefactor of kinetics of the reactants in electrochemistry. Therefore, a surface with higher hydrophobicity is expected to enlarge the prefactor of the kinetics of $\mathrm{N}_{2}$ adsorption and protonation, making it easier for $\mathrm{N}_{2}$ and protonated $\mathrm{N}_{2}$ molecules to penetrate the water layer which leads to a more facile NRR process.

Finally, the free energy pathways of the NRR were calculated at those six studied sites using a computational hydrogen electrode method (Fig. 6c-h).${ }^{57}$ Because the direct activation of $\mathrm{N}-\mathrm{N}$ bonds at the protonated $\mathrm{N}_{2}$-species was found to be difficult on the phosphorene surfaces and the surfaces do not adsorb hydrazine $\left(\mathrm{H}_{2} \mathrm{NNH}_{2}\right)$, only two mechanisms were found
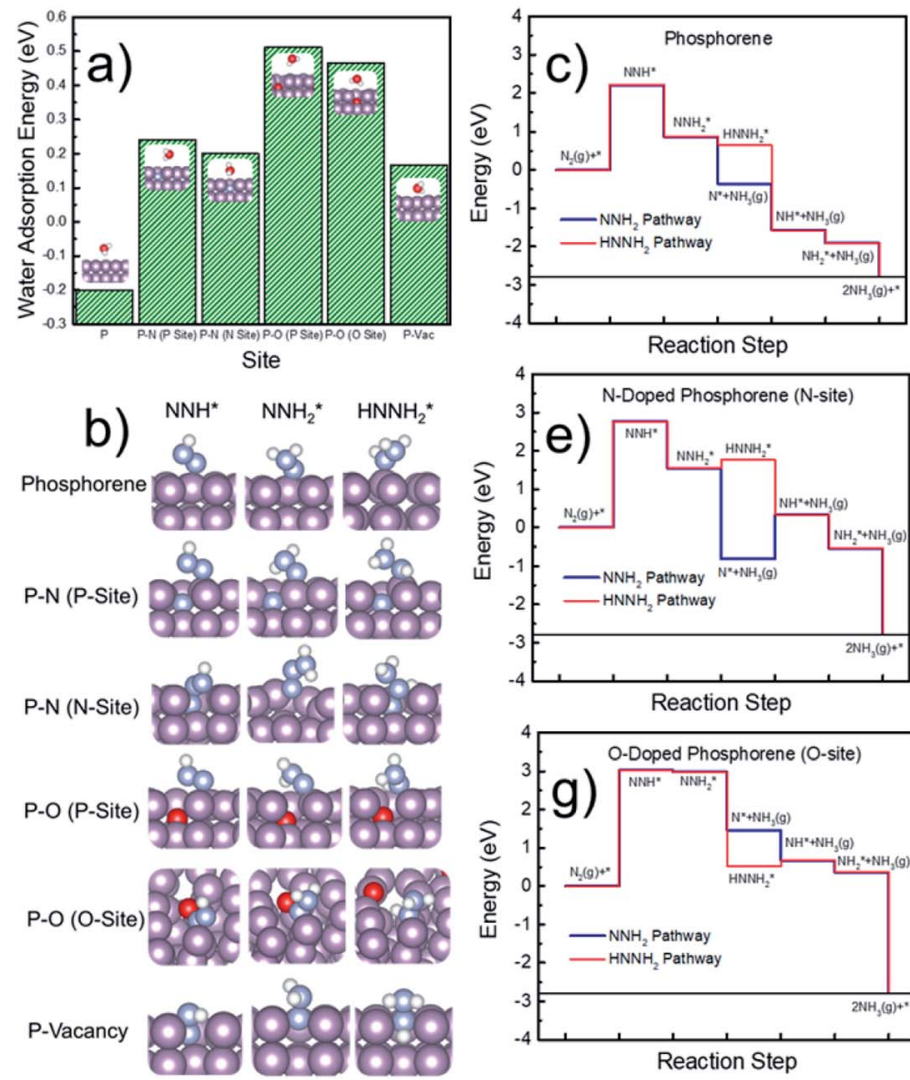
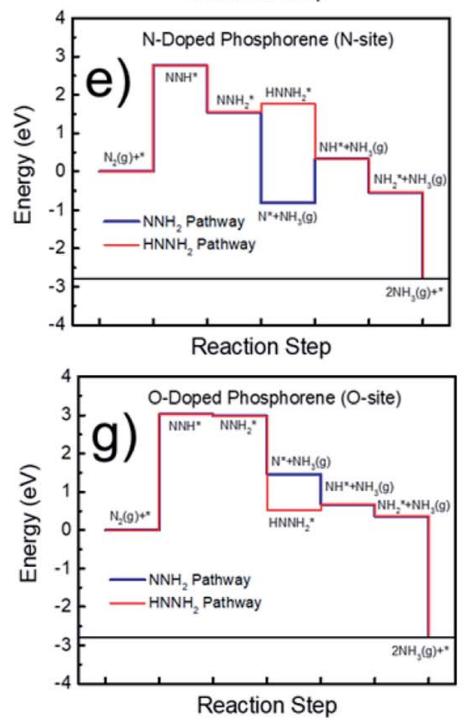
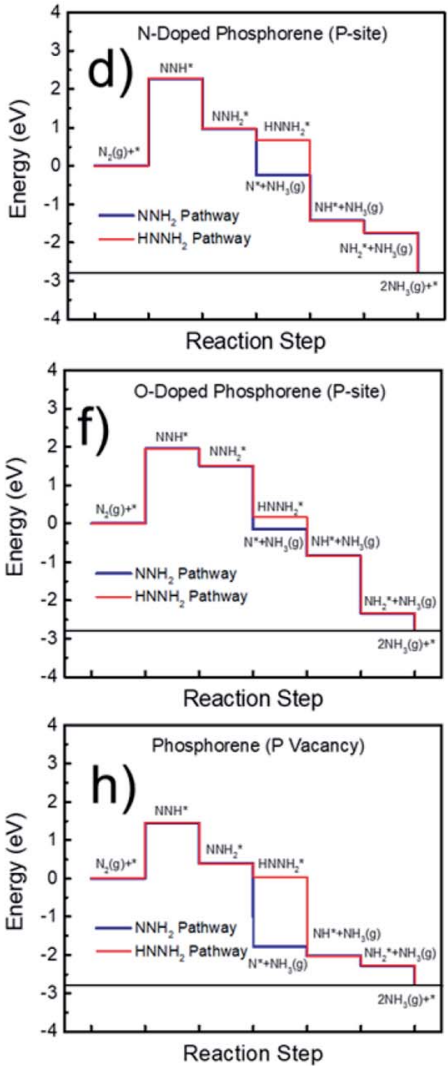

Fig. 6 (a) Calculated adsorption free energy of water at six typical sites on the phosphorene surfaces. Insets show the optimized water adsorption geometries. (b) Optimized structures of $\mathrm{NNH}^{*}, \mathrm{NNH}_{2}^{*}$, and $\mathrm{HNNH}_{2}^{*}$ species adsorbed at the six typical sites. (c-h) Calculated free energy pathways of the NRR at six typical sites on the phosphorene surfaces. Purple, blue, white, and red spheres represent $\mathrm{P}, \mathrm{N}, \mathrm{H}$, and $\mathrm{O}$, respectively. 
to be available at the phosphorene surfaces: the pathways, respectively, through the dissociation of $\mathrm{NNH}_{2}$ and $\mathrm{HNNH}_{2}$. For each pathway, the formation of $\mathrm{NH}_{3}$ gas is considered as the following elementary step after the formation of $\mathrm{NNH}_{2}$ or $\mathrm{HNNH}_{2}$, because the surface does not have a stabilized $\mathrm{NH}_{3}$ adsorption geometry. It can be seen that the potentialdetermining step (PDS) is the direct formation of $\mathrm{NNH}^{*}$ at all the reactive sites. Except for the O-site, all the other sites show more favorable energetics for the $\mathrm{NNH}_{2}$ pathway. The P-vacancy site shows the lowest PDS (Fig. 6h). However, due to the high formation energy of a P-vacancy $(1.03 \mathrm{eV})$, it is expected that this site is not abundant in the system. The P-site at the O-doped phosphorene surface shows the second lowest PDS, suggesting that the electronic effects from the oxygen nearby could improve the NRR activity. Although the P-site in the O-doped phosphorene model has lower potential-determining free energy (Fig. 6f), the O-site in this system has a very high free energy for the formation of $\mathrm{NNH}^{*}$ (Fig. 6g), which is significantly higher than those of all other evaluated sites. Therefore, we conclude that doping with $\mathrm{O}$ makes $\mathrm{P}$-sites more active for the NRR, and it also dilutes the ratio of active sites due to the relatively inert $\mathrm{O}$-sites. In contrast, doping with $\mathrm{N}$ does not lead to a significant up-shift of the reaction free energy as compared to that of the pure phosphorene, and meanwhile, its improved hydrophobicity leads to a more facile NRR process due to the expected larger prefactor in NRR kinetics (Fig. 6a).

Although the formation of $\mathrm{NNH}^{*}$ has relatively high energy, the energetics of this step is electronically tunable and expected to be significantly lowered under applied potentials. It should be noted that these are only the thermodynamic trends for the site-specific NRR activity; we believe that a more complicated model with an explicit solvation model can help to precisely evaluate the free energy of the PDS which can be directly comparable to the experimental overpotentials. However, due to the tremendous computational cost, we only evaluated the trends in the NRR activity in this study. Meanwhile, since P, N, and $\mathrm{C}$ are typical inert $\mathrm{p}$-block elements, we expect that the reaction energy of the PDS is intrinsically higher than that of other transition metal catalysts for the NRR, due to the lack of active electronic structures. Therefore, the hydrophobicity may be the key factor that leads to the relatively high FE in our experimental observations. It can be observed from Fig. 6a that the calculated $\mathrm{P}-\mathrm{O}$ structures have higher hydrophobicity than the P-N structures. We note that according to our XPS (Fig. 2c) and EDX results (Fig. $2 \mathrm{~h}-\mathrm{k}$ ), our $\mathrm{N}$-doped phosphorene has an almost equal amount of $\mathrm{P}-\mathrm{O}$ and $\mathrm{P}-\mathrm{N}$ in the system. Taking this into account, our N-phosphorene system should also have strong hydrophobicity due to the co-existence of both $\mathrm{O}$ and $\mathrm{N}$. With the significant hydrophobic properties after doping with $\mathrm{N}$ and $O$ (Fig. 6a), the NRR is predicted to show a significant FE, in qualitative agreement with our experimental studies.

\section{Conclusions}

We have developed a facile and efficient strategy for preparing $\mathrm{N}$-doped phosphorene nanosheets using oxidized BP to our advantage, providing abundant reactive sites for the doping.
The newly designed N-phosphorene shows an impressive catalytic activity and stability for the NRR process, exhibiting an excellent $\mathrm{NH}_{3}$ yield rate $\left(18.79 \mu \mathrm{g} \mathrm{h}^{-1} \mathrm{mg}_{\mathrm{CAT}}{ }^{-1}\right)$ and $\mathrm{FE}(21.51 \%)$ at a low potential under ambient conditions. DFT calculations indicate that the thermodynamically favorable doping of $\mathrm{N}$ and $\mathrm{O}$ at the phosphorene surface leads to a significant increase of hydrophobicity, which improves NRR efficiency. We believe that the development of heteroatom doped phosphorene opens a promising avenue for metal-free catalyst synthesis, which deserves further research in terms of energy conversion.

\section{Author contributions}

T. M., M. B., Y. C., and J. G. S. conceived and designed the research work. G. X. and H. L. conducted the major experimental work (catalysis) and theoretical calculations, respectively. A. S. R. B., M. B. E., M. J. N., and D. L. made significant contributions in materials preparation, characterization and data analysis. Y. C. and T. M. provided all facilities for catalysis experiments, while J. G. S. and M. B. provided the necessary instruments and facilities for materials synthesis, functionalization and characterization. Y. C., J. G. S., M. B. and T. M. supervised all the research phases. G. X. and M. B. wrote the first draft of the paper. All authors discussed the results and commented on the manuscript.

\section{Conflicts of interest}

There are no conflicts to declare.

\section{Acknowledgements}

This research was sponsored by the Australian Research Council (ARC) through the Discovery Early Career Researcher Award (DE150101306), Linkage Project (LP160100927), Discovery project (DP160101301) and ARC Research Hub for Graphene Enabled Industry Transformation funding (IH150100003), Faculty of Science Strategic Investment Funding 2019 of University of Newcastle, CSIRO Energy, Liaoning Revitalization Talents Program - Pan Deng Scholars (XLYC1802005), Liaoning BaiQianWan Talents Program, the National Science Fund of Liaoning Province for Excellent Young Scholars, Science and Technology Innovative Talents Support Program of Shenyang (RC180166), Fundamental Research Funds for the Central Universities (GK201901002 and 2017TS049), the 111 Project (B14041), and China Scholarship Council. We acknowledge the use of the Queensland node of the Australian National Fabrication Facility (ANFF-Q) and Centre for Microscopy and Microanalysis (CMM) at the University of Queensland. The authors thank Dr Ashley D. Slattery of Adelaide Microscopy at the University of Adelaide for his help with Titan TEM analysis.

\section{Notes and references}

1 M. Batmunkh, M. Bat-Erdene and J. G. Shapter, Adv. Mater., 2016, 28, 8586-8617. 
2 V. Tran, R. Soklaski, Y. Liang and L. Yang, Phys. Rev. B: Condens. Matter Mater. Phys., 2014, 89, 235319.

3 F. Xia, H. Wang and Y. Jia, Nat. Commun., 2014, 5, 4458.

4 L. Li, Y. Yu, G. J. Ye, Q. Ge, X. Ou, H. Wu, D. Feng, X. H. Chen and Y. Zhang, Nat. Nanotechnol., 2014, 9, 372.

5 M. Buscema, D. J. Groenendijk, S. I. Blanter, G. A. Steele, H. S. J. van der Zant and A. Castellanos-Gomez, Nano Lett., 2014, 14, 3347-3352.

6 J. Sun, H.-W. Lee, M. Pasta, H. Yuan, G. Zheng, Y. Sun, Y. Li and Y. Cui, Nat. Nanotechnol., 2015, 10, 980.

7 M. Batmunkh, M. Bat-Erdene and J. G. Shapter, Adv. Energy Mater., 2018, 8, 1701832.

8 K. Zhang, B. Jin, C. Park, Y. Cho, X. Song, X. Shi, S. Zhang, W. Kim, H. Zeng and J. H. Park, Nat. Commun., 2019, 10, 2001.

9 M. Batmunkh, A. Shrestha, M. Bat-Erdene, M. J. Nine, C. J. Shearer, C. T. Gibson, A. D. Slattery, S. A. Tawfik, M. J. Ford, S. Dai, S. Qiao and J. G. Shapter, Angew. Chem., Int. Ed., 2018, 57, 2644-2647.

10 M. Zhu, S. Kim, L. Mao, M. Fujitsuka, J. Zhang, X. Wang and T. Majima, J. Am. Chem. Soc., 2017, 139, 13234-13242.

11 X. Zhu, T. Zhang, Z. Sun, H. Chen, J. Guan, X. Chen, H. Ji, P. Du and S. Yang, Adv. Mater., 2017, 29, 1605776.

12 B. Tian, B. Tian, B. Smith, M. C. Scott, R. Hua, Q. Lei and Y. Tian, Nat. Commun., 2018, 9, 1397.

13 M. Batmunkh, M. Myekhlai, A. S. R. Bati, S. Sahlos, A. D. Slattery, T. M. Benedetti, V. R. Gonçales, C. T. Gibson, J. J. Gooding, R. D. Tilley and J. G. Shapter, J. Mater. Chem. A, 2019, 7, 12974-12978.

14 Z. Yuan, J. Li, M. Yang, Z. Fang, J. Jian, D. Yu, X. Chen and L. Dai, J. Am. Chem. Soc., 2019, 141, 4972-4979.

15 X. Ren, J. Zhou, X. Qi, Y. Liu, Z. Huang, Z. Li, Y. Ge, S. C. Dhanabalan, J. S. Ponraj, S. Wang, J. Zhong and H. Zhang, Adv. Energy Mater., 2017, 7, 1700396.

16 L. Zhang, L.-X. Ding, G.-F. Chen, X. Yang and H. Wang, Angew. Chem., Int. Ed., 2019, 58, 2612-2616.

17 A. Favron, E. Gaufrès, F. Fossard, A.-L. Phaneuf-L'Heureux, N. Y. W. Tang, P. L. Lévesque, A. Loiseau, R. Leonelli, S. Francoeur and R. Martel, Nat. Mater., 2015, 14, 826.

18 M. Bat-Erdene, M. Batmunkh, S. A. Tawfik, M. Fronzi, M. J. Ford, C. J. Shearer, L. Yu, M. Dadkhah, J. R. Gascooke, C. T. Gibson and J. G. Shapter, Adv. Funct. Mater., 2017, 27, 1704488.

19 C. R. Ryder, J. D. Wood, S. A. Wells, Y. Yang, D. Jariwala, T. J. Marks, G. C. Schatz and M. C. Hersam, Nat. Chem., 2016, 8, 597.

20 C. Han, Z. Hu, L. C. Gomes, Y. Bao, A. Carvalho, S. J. R. Tan, B. Lei, D. Xiang, J. Wu, D. Qi, L. Wang, F. Huo, W. Huang, K. P. Loh and W. Chen, Nano Lett., 2017, 17, 4122-4129.

21 Y. Liu, P. Gao, T. Zhang, X. Zhu, M. Zhang, M. Chen, P. Du, G.-W. Wang, H. Ji, J. Yang and S. Yang, Angew. Chem., Int. Ed., 2019, 58, 1479-1483.

22 A. Hashmi and J. Hong, J. Phys. Chem. C, 2015, 119, 91989204.

23 N. Yang, L. Li, J. Li and Z. Wei, Phys. Chem. Chem. Phys., 2019, 21, 4899-4906.
24 X. Xuan, Z. Zhang and W. Guo, Nanoscale, 2018, 10, 78987904.

25 B. Liu, M. Köpf, A. N. Abbas, X. Wang, Q. Guo, Y. Jia, F. Xia, R. Weihrich, F. Bachhuber, F. Pielnhofer, H. Wang, R. Dhall, S. B. Cronin, M. Ge, X. Fang, T. Nilges and C. Zhou, Adv. Mater., 2015, 27, 4423-4429.

26 Y. Xu, J. Yuan, L. Fei, X. Wang, Q. Bao, Y. Wang, K. Zhang and Y. Zhang, Small, 2016, 12, 5000-5007.

27 B. Yang, B. Wan, Q. Zhou, Y. Wang, W. Hu, W. Lv, Q. Chen, Z. Zeng, F. Wen, J. Xiang, S. Yuan, J. Wang, B. Zhang, W. Wang, J. Zhang, B. Xu, Z. Zhao, Y. Tian and Z. Liu, Adv. Mater., 2016, 28, 9408-9415.

28 X. Li, H. Wang, J. T. Robinson, H. Sanchez, G. Diankov and H. Dai, J. Am. Chem. Soc., 2009, 131, 15939-15944.

29 T. Y. Ma, J. Ran, S. Dai, M. Jaroniec and S. Z. Qiao, Angew. Chem., Int. Ed., 2015, 54, 4646-4650.

30 M. Batmunkh, C. J. Shearer, M. J. Biggs and J. G. Shapter, J. Mater. Chem. A, 2016, 4, 2605-2616.

31 I.-Y. Jeon, H.-J. Choi, S.-M. Jung, J.-M. Seo, M.-J. Kim, L. Dai and J.-B. Baek, J. Am. Chem. Soc., 2013, 135, 1386-1393.

32 J. Xu, I.-Y. Jeon, J.-M. Seo, S. Dou, L. Dai and J.-B. Baek, Adv. Mater., 2014, 26, 7317-7323.

33 L. Shao, H. Sun, L. Miao, X. Chen, M. Han, J. Sun, S. Liu, L. Li, F. Cheng and J. Chen, J. Mater. Chem. A, 2018, 6, 2494-2499.

34 M. Bat-Erdene, M. Batmunkh, C. J. Shearer, S. A. Tawfik, M. J. Ford, L. Yu, A. J. Sibley, A. D. Slattery, J. S. Quinton, C. T. Gibson and J. G. Shapter, Small Methods, 2017, 1, 1700260.

35 H. Liu, A. T. Neal, Z. Zhu, Z. Luo, X. Xu, D. Tománek and P. D. Ye, ACS Nano, 2014, 8, 4033-4041.

36 M. Batmunkh, K. Vimalanathan, C. Wu, A. S. R. Bati, L. Yu, S. A. Tawfik, M. J. Ford, T. J. Macdonald, C. L. Raston, S. Priya, C. T. Gibson and J. G. Shapter, Small Methods, 2019, 3, 1800521.

37 H.-K. Jeong, Y. P. Lee, R. J. W. E. Lahaye, M.-H. Park, K. H. An, I. J. Kim, C.-W. Yang, C. Y. Park, R. S. Ruoff and Y. H. Lee, J. Am. Chem. Soc., 2008, 130, 1362-1366.

38 W. Lu, H. Nan, J. Hong, Y. Chen, C. Zhu, Z. Liang, X. Ma, Z. Ni, C. Jin and Z. Zhang, Nano Res., 2014, 7, 853-859.

39 B. Tian, B. Tian, B. Smith, M. C. Scott, Q. Lei, R. Hua, Y. Tian and Y. Liu, Proc. Natl. Acad. Sci. U. S. A., 2018, 115, 43454350 .

40 B. Sa, Y.-L. Li, J. Qi, R. Ahuja and Z. Sun, J. Phys. Chem. C, 2014, 118, 26560-26568.

41 T. Ahmed, S. Balendhran, M. N. Karim, E. L. H. Mayes, M. R. Field, R. Ramanathan, M. Singh, V. Bansal, S. Sriram, M. Bhaskaran and S. Walia, npj 2D Mater. Appl., 2017, 1, 18.

42 J. Wu, X. Zheng, C. Jin, J. Tian and R. Yang, Carbon, 2015, 92, 327-338.

43 J. Sun, G. Zheng, H.-W. Lee, N. Liu, H. Wang, H. Yao, W. Yang and Y. Cui, Nano Lett., 2014, 14, 4573-4580.

44 J. Kang, J. D. Wood, S. A. Wells, J.-H. Lee, X. Liu, K.-S. Chen and M. C. Hersam, ACS Nano, 2015, 9, 3596-3604. 
45 H. Tao, C. Choi, L.-X. Ding, Z. Jiang, Z. Han, M. Jia, Q. Fan, Y. Gao, H. Wang, A. W. Robertson, S. Hong, Y. Jung, S. Liu and Z. Sun, Chem, 2019, 5, 204-214.

46 S. Chen, S. Perathoner, C. Ampelli, C. Mebrahtu, D. Su and G. Centi, Angew. Chem., Int. Ed., 2017, 56, 2699-2703.

47 G.-R. Xu, M. Batmunkh, S. Donne, H. Jin, J.-X. Jiang, Y. Chen and T. Ma, J. Mater. Chem. A, 2019, 7, 25433-25440.

48 J. Wang, L. Yu, L. Hu, G. Chen, H. Xin and X. Feng, Nat. Commun., 2018, 9, 1795.

49 H.-M. Liu, S.-H. Han, Y. Zhao, Y.-Y. Zhu, X.-L. Tian, J.-H. Zeng, J.-X. Jiang, B. Y. Xia and Y. Chen, J. Mater. Chem. A, 2018, 6, 3211-3217.

50 M. Bat-Erdene, G. Xu, M. Batmunkh, A. S. R. Bati, J. J. White, M. J. Nine, D. Losic, Y. Chen, Y. Wang, T. Ma and J. G. Shapter, J. Mater. Chem. A, 2020, 8, 4735-4739.

51 Y. Zheng, Y. Jiao, M. Jaroniec and S. Z. Qiao, Angew. Chem., Int. Ed., 2015, 54, 52-65.
52 H. Li, K. Shin and G. Henkelman, J. Chem. Phys., 2018, 149, 174705.

53 B. Yu, H. Li, J. White, S. Donne, J. Yi, S. Xi, Y. Fu, G. Henkelman, H. Yu, Z. Chen and T. Ma, Adv. Funct. Mater., 2020, 30, 1905665.

54 J. Xu, A. Avellan, H. Li, X. Liu, V. Noël, Z. Lou, Y. Wang, R. Kaegi, G. Henkelman and G. V. Lowry, Adv. Mater., 2020, 32, 1906910.

55 Z. Cao, H. Li, X. Xu and J. Xu, Chem. Eng. J., 2020, 394, 124876.

56 V. Tripkovic and T. Vegge, J. Phys. Chem. C, 2017, 121, 2678526793.

57 J. K. Nørskov, J. Rossmeisl, A. Logadottir, L. Lindqvist, J. R. Kitchin, T. Bligaard and H. Jónsson, J. Phys. Chem. B, 2004, 108, 17886-17892. 